\title{
Evolução dos níveis de beta-hCG após tratamento sistêmico da gravidez ectópica íntegra
}

\author{
J. ELITO J UNIOR, M. UCHIYAMA, L. CAMANO \\ Disciplina de Obstetrícia da Universidade Federal de São Paulo - Escola Paulista de Medicina, São Paulo, SP.
}

RESUMO - ОвJ ETIVo. O tratamento sistêmico da gravidez ectópica (GE) íntegra com metotrexato (MTX) tornou-se conduta alternativa para tradicional atitude cirúrgica. 0 acompanhamento dos casos é realizado com dosagens seriadas de betahCG, já que a imagem ultra-sonográfica persiste por tempo prolongado, sendo um parâmetro ruim de seguimento. 0 objetivo do nosso estudo é de avaliar o comportamento da curva de beta-hCG após a ministração do medicamento.

Pacientes e Método. Foram incluídas neste estudo pacientes estáveis hemodinamicamente, com massa anexial menor ou igual a $4,5 \mathrm{~cm}$, sem sinais de doença hepática, renal ou supressão da medula óssea. $O$ tratamento foi feito com MTX $50 \mathrm{mg} / \mathrm{m}^{2}$ I M (dose única) em regime de internação. Vinte e quatro pacientes fizeram parte deste trabalho e foram acompanhadas com dosagens de beta-hCG nos dias 1, 4 e 7 pós-MTX. Casos em que os títulos de beta-hCG caíram acima de 15\% entre os dias 4 e 7 receberam alta hospitalar e eram acompanhados semanalmente até títulos negativos. Por outro lado, quando esta queda era inferior a $\mathbf{1 5 \%}$, essas pacientes receberam nova dose de MTX.

Resultados. Doze (50,0\%) dos 24 casos tiveram

\section{INTRODUÇÃO}

A gravidez ectópica (GE) é consi derada uma doença de grande importância, por várias razões. Entre elas citamos o número crescente de manifestações, por vezes de prognóstico ominoso, e a perspectiva atual de um possível diagnóstico precoce e da realização de condutas conservadoras.

Com a oportunidade de realizar o tratamento na sua fase precoce, a conduta cirúrgica tradicional para GE pode ser substituída pel o tratamento sistêmico com MTX, nas pacientes que desejam preservar sua função reprodutiva.

O diagnóstico de GE é realizado precocemente, na atualidade, graças aos avanços da tecnologia ecográfica e dos recursos laboratoriais. Assim, destacamos o desenvol vimento de técnicas imunoenzi máticas, radioimunológicas e, mais recentemente, imu- aumento dos títulos de beta-hcG nos primeiros quatro dias após a injecão IM de MTX, oito (33,3\%) apresentaram queda paulatina dos valores e em quatro $(16,7 \%)$ casos os valores de beta-hCG foram negativos no quarto dia pós-MTX. Apenas duas pacientes $(8,3 \%)$ necessitaram de segunda dose de MTX por não ter havido queda dos títulos de betahCG superior a $15 \%$ entre o quarto e o sétimo dia.

Conclusões. A elevação dos títulos de beta-hCG, entre o momento da ministração do MTX e o quarto dia após a medicação, foi evento freqüente $(50,0 \%)$. A queda de mais de $15 \%$ dos valores de beta-hCG, apurada no quarto e no séti mo dia pósMTX, constitui parâmetro importante para o critério de alta hospitalar. Por outro lado, redução menor que $15 \%$ dos níveis séricos de beta-hCG representa indicação para ministração de nova dose de MTX. Em apenas duas pacientes $(8,4 \%)$ houve necessidade de segunda dose de MTX, pois os títulos de beta-hCG, avaliados no quarto e no sétimo dia após o MTX, apresentaram queda, porém, inferior a $\mathbf{1 5 \%}$.

UNITERMOS: Gravidez ectópica. Beta-hCG. Metotrexato.

norradiométricas ou imunoenzimomimétricas, que utilizam anticorpos monoclonais para aumentar a sensibilidade e a especificidade das dosagens da subunidade beta do hormônio gonadotrópico coriônico (beta-hcG), segundo Fernandez $\&$ Bourget ${ }^{1}$.

$\mathrm{Na}$ gravidez ectópica, a quantidade de beta-hcG aparece diminuída, em relação àquela das gestações intra-uterinas. Em gestações normais, o betahcG sérico dobra de val or a cada 48 horas, enquanto nas gravidezes inviáveis (abortamento ou GE) o aumento, neste período de 48 horas, é de $66 \%^{2,3}$.

O acompanhamento dos casos tratados com MTX é realizado com dosagens seriadas de beta-hcG quantitativo, já que a imagem ultra-sonográfica persiste por tempo prolongado, sendo um parâmetro ruim de seguimento ${ }^{4,5}$. Os títulos de betahCG, portanto, são importantes, tanto no momento do diagnóstico de GE, quanto no acompanhamento 
dos casos. O objetivo do nosso estudo é de avaliar o comportamento da curva de beta-hcG após a ministração do medicamento.

\section{PACIENTES E MÉTODO}

E ste estudo prospectivo foi realizado na Disciplina de Obstetrícia da Universidade F ederal de São Paulo (Escola Paulista de Medicina), em 24 pacientes, no período de abril de 1994 a agosto de 1995.

A atenção do nosso estudo esteve vol tada inicialmente à realização do diagnóstico precoce de GE íntegra incipiente. Com dados positivos na história clínica, valorizam-se as pacientes consideradas de risco para GE, ou seja, as que tenham antecedentes de doença inflamatória pélvica, cirurgia tubária e GE prévia, e as que apresentam dor abdominal ou pélvica, atraso menstrual com sangramento genital. Ao exame ginecológico, ressaltamos o encontro de tumoração látero-uterina ou dor em região anexial.

A propedêutica foi complementada com sol icitação de us transvaginal e dosagem sérica de beta-hcG.

À luz da us transvaginal, visibilizamos o útero vazio com massa anexial complexa, distinta dos ovários, que pode, por vezes, conter o saco gestacional sem ou com embrião, e este com possíveis bati mentos cardíacos. E m al gumas si tuações o exame não elucida o diagnóstico, basicamente quando não houver massa anexial ou gravidez intra-uterina detectável.

Realizou-se, a seguir, dosagem da subunidade beta do hormônio gonadotrópico coriônico, descartando-se o diagnóstico de GE em presença de resultados negativos. Quando os valores foram superiores a $1.500 \mathrm{mu} / \mathrm{mL}$, e a us transvaginal não visibilizou gravidez tópica, confirmamos, por meio do diagnóstico algorítmico, a existência de uma GE, segundo a padronização deStovall et al . ${ }^{6}$ No entanto, pacientes com títulos inferiores àquele foram seguidas com dosagem a cada 48 horas, além de serem acompanhadas com a us transvaginal, tendo por objetivo excluir eventual abortamento incompleto.

Firmado o diagnóstico de GE íntegra, obedecemos a alguns critérios, antes de iniciar a terapêutica com MTX.

Os critérios de incl usão dos casos foram a estabilidade hemodinâmica e o diâmetro máximo da massa anexial igual ou menor que $4,5 \mathrm{~cm}$. A paciente também precisou manifestar o desejo de gravidez futura e concordar em participar do estudo, após consentimento escrito, em documento formulado e aprovado pela Comissão de Ética Médica da Universidade F ederal de São Paulo. Outrossim, as pacientes foram informadas da possibilidade de falha do tratamento, que pode ocorrer em 4 a $5 \%$ dos $\operatorname{casos}^{7,8}$.
F oram excluí das do tratamento medicamentoso da GE íntegra incipiente as pacientes necessitadas de hemotransfusão, reconhecida sensibilidade ao MTX, evidências de doença hepática, renal ou supressão da medula óssea. Não fizeram parte do protocolo as pacientes com discrasias sanguíneas, como leucopenia (número de glóbulos brancos menor que $2.000 \mathrm{cel} . / \mathrm{cm}^{3}$ ) e trombocitopenia (número de plaquetas menor que 100.000). Também não participaram do protocolo as que apresentavam transaminases alteradas ou doença renal (creatinina mai or que $1,5 \mathrm{mg} / \mathrm{dL}$ ).

Os casos incluídos no protocolo do nosso estudo internaram-se na Disci plina de Obstetrícia da U niversidade F ederal de São Paul o (E scola Paulista de Medicina) e foram tratados inicialmente com ministração de dose única de MTX $\left(50 \mathrm{mg} / \mathrm{m}^{2}\right.$ por via intramuscular), e complementado com nova dose, quando indicado.

O acompanhamento se fez por meio de dosagens de beta-hcG, realizadas imediatamente antes da ministração do MTX, no quarto e séti mo dias após o emprego desta droga. As pacientes com queda dos títulos de beta-hcG acima de 15\%, apurada no 4ํ e no 70 dia, tiveram alta hospitalar e seguimento ambulatorial com dosagens semanais de beta-hcG, até a queda de valores a níveis pré-gravídicos. Quando a queda foi menor que $15 \%$, no séti mo dia após o emprego do MTX, ministrou-se nova dose, em regime de internação, seguindo a mesma sistemati zação predita ${ }^{6,9,10}$.

Os dados ultra-sonográficos não foram utilizados como critério de alta, já que a imagem us pode persistir por algum tempo, mesmo após o declínio dos valores de beta-hcG ${ }^{4,5}$.

O critério de insucesso na ministração de MTX, na GE íntegra, foi baseado na persistência de el evados níveis de beta-hcG, após a segunda dose de MTX ou no início de sinais e sintomas clássicos de ruptura tubária consumada, apurados pela clíni ca e pel a US transvaginal. Nessa oportunidade foi realizada a laparotomia.

Após a alta hospitalar, as pacientes foram seguidas em ambulatório personalizado, semanalmente, realizando-se avaliação clínica e dosagem de beta-hcG.

Considerou-se como critério de sucesso do tratamento a obtenção de títulos de beta-hcG inferiores a $25 \mathrm{mul} / \mathrm{mL}$.

\section{RESULTADOS}

Avaliamos a variação dos valores de beta-hcG entre o primei ro e o quarto dia após a ministração do MTX, e observamos se ocor reu el evação, queda ou 
Tabela 1 - Variação dos títulos de beta-hCG entre o primeiro e o quarto dia após a ministração do MTX

\begin{tabular}{|lcc|}
\hline \multicolumn{1}{|c|}{ Variação } & № & $\%$ \\
Elevação & 12 & 50,0 \\
Queda & 8 & 33,3 \\
Abaixo de $25 \mathrm{mUI} / \mathrm{mL}$ & 4 & 16,7 \\
Total & 24 & 100,0 \\
\hline
\end{tabular}

Tabela 2 - Variação da porcentagem dos títulos de betahCG, entre o quarto e o sétimo dia após a ministração do MTX

$\begin{array}{ccc}\text { Variação (\%) } & \text { № } & \% \\ \geq 15 & 12 & 85,7 \\ <15 & 2 & 14,3 \\ \text { Total } & 14 & 100,0\end{array}$

* Foram excluídos os casos com títulos de beta-hCG abaixo de $25 \mathrm{mUl} / \mathrm{mL}$ no quarto dia após o MTX (4 casos), e os que evoluíram para laparotomia (6 casos).

Tabela 3 - Necessidade do emprego da segunda dose de MTX

\begin{tabular}{|ccc|}
\hline Necessidade & № & $\%$ \\
Não & 22 & 91,6 \\
Sim & $2^{*}$ & 8,4 \\
Total & 24 & 100,0 \\
\hline
\end{tabular}

Tabela 4 - Tempo necessário para regressão dos títulos de beta-hCG a níveis pré-gravídicos, nos casos de sucesso do tratamento

\begin{tabular}{|crc|}
\hline Tempo em dias & № & $\%$ \\
$<15$ & 9 & 50,0 \\
$15 \vdash 30$ & 7 & 38,8 \\
$>30$ & 2 & 11,2 \\
Total & 18 & 100,0 \\
\hline Média $=17,6 \pm 12,7$ dias. & & \\
\hline
\end{tabular}

se os títulos se tornaram negativos, comorepresentado na tabela 1.

F oi analisada, também, a variação da porcentagem dos títulos de beta-hcG, entre o quarto e o séti mo dia após a mi nistração do MTX, e verificamos se esta foi igual ou superior a $15 \%$ ou se inferior a $15 \%$, conforme demonstra a tabela 2 .

As pacientes que apresentaram queda dos títulos de beta-hcG inferior a 15\%, entre os dias 4 e 7 após a ministração do MTX, receberam uma segunda dose de MTX, como representado na tabela 3.

Outro resul tado que obtivemos, na aval iação dos títulos de beta-hcG, foi o tempo necessário para regressão dos valores de beta-hcG a níveis pré- gravídicos, nos casos de sucesso do tratamento, conforme dados apresentados na tabela 4.

\section{DISCUSSÃO}

É de grandevalia saber interpretar os valores de beta-hcG. Fernandez \& B ourget $^{1}$, num estudo com 95 pacientes submetidas a tratamento medi camentoso para GE, elaboraram uma curva que demonstra o comportamento do beta-hcG, após o tratamento. O porcentual, de $100 \%$, foi estabelecido como o valor inicial de beta-hcG, antes de ser iniciado o tratamento com MTX. É interessante observar que, na primeira semana, há um aumento nos níveis de beta-hcG, que só retornam ao valor inicial (100\%) após cerca de oito dias. Esta el evação pode chegar até $25 \%$ do valor inicial e se deve a dois fatores: à aceleração inicial do metabolismo do beta-hcG pelo MTX, e à destruição trofoblástica celular que aumenta o pool sistêmico․

O conhecimento dessa evolução inicial da concentração do beta-hcG evita intervenções desnecessárias, na primeira semana pós-tratamento. No caso de não haver declínio na concentração de betahCG, após a primeira semana, ou de a evolução diferir da curva apresentada, pode-se adicionar mais uma injeção IM de MTX ${ }^{1}$.

Segundo os mesmos autores, o retorno aos valores pré-gravídicos $(<10 \mathrm{mul} / \mathrm{mL})$ do beta-hcG ocorre em cerca de 30 dias. Ressaltam que é importante realizar uma dosagem controle no $2^{\circ}, 5^{\circ}$ e $10^{\circ}$ dias após a mi nistração da droga e depois, semanalmente, até o retorno aos valores pré-gravídicos.

E m nosso trabal ho, seguimos essa linha de raciocíni o com al gumas modificações. Com o diagnóstico de GE, dosamos o beta-hcG antes de ministrar o MTX, e repetimos a operação no quarto e no séti mo dia após o início da medicação. Realizamos dosagens semanais, até val ores inferiores a $25 \mathrm{mul} / \mathrm{mL}$.

Observamos a variação dos títulos de beta-hcG entre o primeiro e o quarto dia após a ministração do MTX (tabela 1), verificando que, em $50,0 \%$ dos casos, ocorreu elevação dos títulos; $33,3 \%$ deles apresentaram queda dos valores e em $16,7 \%$ das pacientes houve retorno dos títulos de beta-hcG a valores pré-gravídicos.

A el evação dos títulos entre o primei ro e o quarto dia ocorreu em 50,0\% dos casos, sendo um evento freqüente não significando, necessariamente, má evolução do caso, pois metade dessas pacientes $(25,0 \%)$ evoluiu com sucesso e as outras $25,0 \%$ foram submetidas a laparotomia, por apresentarem al terações clíni cas e propedêuticas que confirmavam ruptura tubária.

E m seis casos (33,3\%) do nosso protocolo (tabela 
1), não ocorreu elevação dos títulos de beta-hcG entre o primeiro e o quarto dia após O MTX. No entanto, houve uma regressão paulatina, retornando aos valores pré-gravídicos, em média, de 14 a 21 dias.

Ocorreu queda rápida dos valores após o MTX, com títulos inferiores a $25 \mathrm{mul} / \mathrm{mL}$, no quarto dia após o medicamento (tabela 1), em 16,7\% dos casos.

Segundo Stovall et al. ${ }^{9}$, um dos critérios mais importantes para saber se um caso está evoluindo bem, após o tratamento com o MTX, é avaliar a variação da porcentagem dos títulos de beta-hcG no quarto e no sétimo dia após a ministração da droga. Se essa variação, em porcentagem, superar os $15 \%$, provavelmente haverá evolução satisfatória. Esse dado foi por nós utilizado como critério de alta. Por outrolado, quando a queda dos títulos de beta-hcG, no quarto eno sétimo dia pós-MTX, foi inferior a 15\%, as pacientes receberam nova dose de MTX. E m nosso estudo, excluímos dessa análise o grupo que teve redução dos títulos de beta-hcG a níveis pré-gravídicos, no quarto dia (4 casos), e os que evoluíram para laparotomia (6 casos). Observamos, na tabela 2 , que $85,7 \%$ dos casos apresentaram queda superior a $15 \%$, nesse período, dos quais apenas duas pacientes $(14,3 \%)$ demonstraram decréscimo do beta-hCG inferior a 15\% entre o quarto e sétimo dias pós-MTX, necessitando de nova dose da droga.

Dessa forma (tabela 3), apenas $8,4 \%$ dos casos receberam a segunda dose de MTX. Em ambos os casos, ocorreu boa evolução após o medicamento, sendo atingi do o sucesso nessas duas situações.

O tempo em dias, para o retorno dos títulos de beta-hcG a valores pré-gravídicos, foi também avaliado (tabela 4), sendo dividido em três grupos: menos que 15 dias, entre 15 e 30 , eacima de 30 dias.

$\mathrm{Na}$ análise dessa variável, somente participaram os casos que ti veram sucesso com o tratamento (75,0\%). Observamos que, na maioria das pacientes, ocorreu queda dos títulos de beta-hcG abaixo de $25 \mathrm{mul} / \mathrm{mL}$, num período inferior a 15 dias $(50,0 \%)$, e entre 15 e 30 dias o retorno aos valores pré-gravídicos ocorreu em $38,8 \%$ dos casos. Apenas em duas situações demoraram mais de 30 dias $(11,2 \%)$. Nestas, houve necessidade de empregar duas doses de MTX, e os títulos se reduziram, em uma delas, após 48 dias da primeira dose. O tempo médio para o retorno dos títulos de beta-hcG a níveis pré-gravídicos foi de 17,6 dias.

\section{CONCLUSÕES}

A elevação dos títulos de beta-hCG, entre o momento da mini stração do MTX e o quarto dia após a medicação, foi evento freqüente $(50,0 \%)$.
A queda de mais de $15 \%$ dos valores de betahcG, entre o quarto e o sétimo dia pós-MTX, constitui parâmetro importante para o critério de alta hospitalar. Por outrolado, redução menor que $15 \%$ dos níveis séricos de beta-hcG representa indicação para ministração de nova dose de MTX.

Em apenas duas pacientes $(8,4 \%)$ houve necessidade de segunda dose de MTX, pois os títulos de beta-hcG, entre o quarto e o sétimo dia após o MTX, apresentaram queda, porém, inferior a 15\%.

\section{SUMMARY}

\section{Evolution of beta-hCG titers after treatment with methotrexate in unruptured ectopic pregnancy}

Овлестіve. The follow-up of this treatment is done by monitorization of beta-hcG titers. The objective of this study is to evaluate the beta-hcG titers after treatment with MTX.

MeTHODS. Twenty four women were included in the study. The inclusion criteria were: ectopic pregnancy $\leq 4.5 \mathrm{~cm}$, beta-hcG $\leq 15.000 \mathrm{mIU} / \mathrm{mL}$, desire of future pregnancy and a written permissi on to participate in the study. All patients were treated with a single dose of methotrexate $\left(50 \mathrm{mg} / \mathrm{m}^{2} \mathrm{IM}\right)$. Patients weremonitored with beta-hc $\mathrm{G}$ titers on days 1,4 and 7 after the MTX injection, and then weekly until the beta-hcG was less than $25 \mathrm{mIU} / \mathrm{mL}$.

RESULTS. The variation of the titers of beta-hCG between day 1 and day 4 after MTX was the following: increase $50,0 \%$, decrease $33.3 \%$ and in levels less than $25 \mathrm{mul} / \mathrm{mL}$ in $16.7 \%$ of the cases. The variation of the titers of beta-hcG between day 4 and day 7 after MTX was the following: decline $\geq$ $15 \%$ in $85.7 \%$ of the cases, and decline $<15 \%$ in $14.3 \%$. There was the necessity of a second dose of MTX in only two cases (8.4\%), since the levels of beta-hcG declined less than $15 \%$, in this period.

CONCLUSION. The titers of beta-hCG increase in $50.0 \%$ of the cases, so it is a frequent event. The understanding of this evolution of beta-hcG titers avoids surgery in the first week of the treatment. [Rev Ass Med Brasil 1998; 44(1): 11-5.]

Key Words: Ectopic pregnancy. Beta-hCG. Methotrexate.

\section{REFERÊNCIAS BIBLIOGRÁFICAS}

1. Fernandez H, Bourget P. Medical treatment of ectopic pregnancy. Contracept Fertil Sex 1992; 20: 511-9.

2. Kamrava M, Taymor M, Berges M. Disappearance of human chorionic gonadotropin following removal of ectopic pregnancy. Obstet Gynecol 1983; 62: 486-90.

3. Steir J, Bergspo P, Myking O. Human chorionic gonadotropin in maternal plasma after induced abortion, spontaneous abortion, and removal of P.E. Obstet Gynecol 1984; 64: 391-7. 
4. Brown DL, Felker RE, Stovall TG, Emerson DS, Ling FW. Serial endovaginal sonography of ectopic pregnancies treated with methotrexate. Obstet.Gynecol 1991; 77: 406-9.

5. Atri M, Bret PM, Tulandi T, Senterman MK. Ectopic pregnancy: evolution after treatment with transvaginal MTX. Obstet Ultrassound 1992; 185: 749-53.

6. Stovall TG, Ling FW. Single dose of methotrexate: an expanded clinical trial. Am J Obstet Gynecol 1993; 168: 1.759-65.

7. Stovall TG, Ling TW, Gray LA. Methotrexate treatment of unrupture ectopic pregnancy. A report of 100 cases. Obstet Gynecol 1991; 77: 749.

8. Stovall TG, Ling TW, Gray LA. Single dose methotrexate for treatment of ectopic pregnancy. Obstet Gynecol 1991; 77: 754-7.

9. Stovall TG, Ling FW. Some new approaches to ectopic pregnancy. Contemp Obstet Gynecol 1992; 37: 35-41.

10. Stovall TG, Ling FW. Ectopic pregnancy: diagnostic and therapeutic algorithms, minimizing surgical intervention. J Reprod Med 1993; 38: 807-11. 